\title{
Coyuntura económica y voto en España, 1985-1996*
}

\author{
Agustí Bosch \\ Universitat Autònoma de Barcelona \\ agusti.bosch@uab.es
}

\section{Clara Riba}

Universitat Pompeu Fabra

clara.riba@upf.edu

\section{Resumen}

El artículo presenta un modelo de popularidad para el Gobierno español entre 1985 y 1996. El modelo se ha elaborado con datos trimestrales procedentes de encuestas del CIS y de la UE y datos económicos procedentes de la OCDE y del INE. Los resultados enfatizan la gran influencia que tuvieron algunas variables de la coyuntura macroeconómica sobre la popularidad del Gobierno socialista, pero sobre todo la influencia que ejercieron las percepciones sobre esta coyuntura macroeconómica. Además, también se revelan las influencias de algunas variables políticas: el carisma del presidente, la adhesión a la CE, la guerra de Kuwait y el escándalo Filesa.

Palabras clave: elecciones, comportamiento electoral, economía, elección racional, estadística, series temporales.

\section{Abstract. Economic situation and voting in Spain, 1985-1996}

The article shows a function of popularity for the Spanish government between 1985 and 1996. The model was developed with quarterly data from opinion surveys (CIS and EU) and from economic series (OECE and INE). The results emphasize several great influences over the popularity of the socialist government: the influence of the macroeconomic situation, the influence of the perceptions about that situation, and the influence of some political variables such as the president's charisma, the joining of the EC, the war in Kuwait, and the Filesa scandal.

Key words: economy and vote in Spain, economic voting. popularity functions, economy and politics.

* Este artículo ha sido escrito en el contexto del proyecto de investigación de la Comisión Interministerial de Ciencia y Tecnología con referencia CICYT SEC96-0510, que también contó con financiación del Institut de Ciències Polítiques i Socials (ICPS). En este proyecto participaron — además de los autores_ Aida Díaz, Maria Ferrer, Joan Font, Jordi Sánchez y Josep M. Vallès, éste último como investigador principal. Versiones preliminares del mismo fueron presentadas en la Conferencia del ICPS sobre Funciones de Popularidad (Barcelona, 20 y 21 de noviembre de 1998) y en el XI Seminario de Investigaciones Políticas y Sociológicas de AEDEMO (Bellaterra, 14 de enero de 2000). 


\section{Sumario}

1. Introducción

2. Datos y metodología

3. Panóramica de resultados

4. Resultados para la componente económica

5. Resultados para la componente
6. Resultados para la componente política

7. Variables descartadas

8. Conclusiones

Bibliografía estructural

\section{Introducción}

Nadie duda de la influencia que tiene la situación económica de un país en la popularidad de la que gozan sus gobernantes. La satisfacción con que los miembros de los gobiernos acogen a los indicadores que señalan una buena salud de la economía nacional, la visión mucho más crítica que manifiesta la oposición hacia estos mismos resultados y la importancia que se les da en los medios de comunicación lo muestran claramente. Pero si bien esta apreciación es algo común en la ciudadanía, en el mundo académico el interés se ha centrado, desde hace más de cincuenta años, en entender cuál es y cómo se estructura esta relación entre economía y popularidad gubernamental, relación que es importante conocer, puesto que las posibilidades de reelección del ejecutivo dependen en gran medida de dicha popularidad. Actualmente, la literatura referente a este tema es amplia, y los investigadores utilizan modelos cada vez más sofisticados para contrastar las distintas teorías que existen al respecto.

En términos generales, la mayor parte de los estudios empíricos, realizados con datos de distintos países, confirman la llamada hipótesis de la responsabilidad, según la cual los electores responsabilizan al Gobierno por la situación económica del país y actúan en consecuencia (Nannestad y Paldam, 1994). Así, premian al Gobierno cuando la situación es buena, dando su voto al partido en el poder, y lo castigan cuando la situación es mala, negando el voto al partido gobernante. Algunos estudios, sin embargo, muestran que el modelo de premio-castigo no acaba de funcionar en situaciones en las que no existe una clara atribución de responsabilidades hacia los partidos que gobiernan.

En Bosch, Díaz y Riba (1999) se realiza un repaso a la literatura sobre funciones de popularidad, con una exposición de las características de los modelos utilizados más habitualmente y de sus principales resultados, y con una extensa discusión de los debates sustantivos que hoy en día el tema tiene planteados. Los trabajos seminales son Mueller (1970), Goodhart y Bhansali (1970) y Kramer (1971), aunque los orígenes del voto económico se hallan en Schumpeter (1945) y Downs (1957). Los debates más interesantes se encuentran en los trabajos de Fiorina (1981), que establece la preponderancia del voto 
retrospectivo frente al prospectivo; Kinder y Kieweit (1981), que defiende la tesis del voto sociotrópico frente al egotrópico; Nannestad y Paldam (1997), que muestra la existencia de ambos tipos de comportamiento en distintos contextos sociales; Sanders, Ward y Marsh (1987), que defiende la influencia de las percepciones subjetivas de la economía frente a la de la coyuntura económica objetiva; Hibbs (1982), que presenta evidencias de valoración de las condiciones económicas a largo plazo, frente a la hipótesis predominante de «miopia»; Clarke y Steward (1995), que ponen el énfasis en las aportaciones personales del líder, y Anderson (1995), que introduce los efectos del contexto. Finalmente, Lewis-Beck (1988) y Norpoth, Lewis-Beck y Lafay (1991) son estudios comparados de referencia obligada.

Aunque se han estimado funciones de popularidad en un gran número de países que celebran regularmente elecciones democráticas, los estudios que han hecho referencia al caso español son más bien escasos. El primer autor en estimar una función de popularidad para España es Amor Bravo (1985, 1987 y 1990), pero sus modelos no son satisfactorios, puesto que en algunos casos obtiene coeficientes positivos para el desempleo y la inflación, contradiciendo tanto la teoría como los resultados empíricos existentes. Posiblemente ello esté relacionado con algunos aspectos derivados de la juventud de la democracia española en la primera parte del período analizado: sistema de partidos no consolidado, prioridades políticas más que económicas o importancia del factor liderazgo. Mancha Navarro (1993) estima posteriormente una serie de modelos para el período comprendido entre 1979 y 1989. Sus resultados tampoco se ajustan correctamente a las previsiones teóricas, puesto que obtiene un signo positivo en el coeficiente estimado de la variable desempleo. Una primera interpretación para esta anomalía es de tipo económico: después de una fase de ajuste duro entre 1982 y 1985 destinada a combatir la crisis, la economía española mejoró ostensiblemente, dando la impresión de buena gestión gubernamental, a pesar del mantenimiento de altas tasas de paro. Una segunda interpretación es de tipo político: quizá la hipótesis de la responsabilidad no funciona en el caso español porque los ciudadanos de este país practican el llamado «policy-oriented voting», es decir, votan en función de la reputación que cada partido tiene para resolver cada tipo de problema. En una situación con altos niveles de desempleo, los electores se decantarían mayoritariamente por votar al partido socialista, que se supone es el partido más interesado y más capacitado para solucionar los problemas del paro.

La escasez de estudios que estimen funciones de popularidad para España hasta principios de los años noventa se ha visto compensada por el interés que han suscitado recientemente todos los aspectos relacionados con el voto económico. En efecto, durante los últimos quince años se han publicado trabajos de varios autores españoles, los cuales no se han limitado a trabajar con datos agregados, sino que han analizado la relación entre economía y voto con datos individuales, hecho que les ha permitido contrastar hipótesis causales que difícilmente podrían plantearse con modelos agregados. Así, Maravall 
y Pzrewoski (1999) muestran que la lógica del voto económico es mucho más compleja de lo que parece. Según esos autores, mediante mecanismos «intertemporales», «exonerativos» y "de oposición», los votantes se alejan con frecuencia de la hipótesis del reward-punishment. Fraile (2001, 2002a) estima modelos individuales de intención de voto en los que incluye variables referentes al contexto político y social además de las variables económicas. Dicha autora aporta evidencias a favor del voto retrospectivo en España durante los años noventa y sostiene que el Gobierno socialista consiguió mantenerse en el poder en 1993, en plena recesión económica, gracias a las políticas sociales implementadas en el pasado. Fraile (2002b) aporta también evidencia de la existencia de voto económico en las elecciones generales del año 2000, cuando la buena situación monetaria, así como por las expectativas generadas por la gestión realizada por el Partido Popular, jugaron a favor de la reelección del Gobierno. Por otra parte, Díaz (1999), Díaz y Riba (1999) y Riba y Díaz (2002) han trabajado con datos de una parte de la realidad española. Díaz ha elaborado una función de popularidad para el Gobierno autonómico de Cataluña; Díaz y Riba han analizado el voto económico en contextos de gobiernos multinivel, donde la atribución de responsabilidades aparece difusa, y Riba y Díaz, con datos individuales, han profundizado en la relación causal que conecta la valoración de las políticas del Gobierno con la aprobación de su gestión y con la intención de voto, mostrando que la aprobación se traduce en intención de voto si supera un determinado umbral que depende de las características de las personas. Barreiro (1999) ahonda en el tema de atribución de responsabilidades al Gobierno. Sánchez-Cuenca y Barreiro (2000) evalúan el impacto de la valoración de la gestión del ejecutivo en el comportamiento electoral y la actuación esperada de otros partidos en la oposición.

El trabajo que se presenta en este artículo es el resultado de la estimación de un modelo de popularidad para el Gobierno español durante casi toda la etapa en la que éste estuvo en manos del partido socialista y bajo la presidencia de Felipe González. Es, por lo tanto, un modelo para España en un período que ya no es de transición, con un sistema de partidos ya consolidado, siempre con el mismo partido en el gobierno, administrando en solitario y con el mismo presidente. Si bien la serie de observaciones de la que se dispone no es tan extensa como sería de desear, es suficiente para intentar modelar una función de popularidad obviando los problemas que habían aparecido en algunos de los estudios para el caso español realizados anteriormente.

A continuación se exponen, en primer lugar, la metodología y los datos utilizados. Seguidamente, se presentan los resultados obtenidos en la estimación del modelo, comentando los coeficientes obtenidos para las variables de cada uno de los componentes de la función: la económica, la estructural y la política. Finalmente, se detallan cuales han sido las variables inicialmente tomadas en cuenta y los motivos por los cuales fueron descartadas. El artículo finaliza con un breve comentario resumen de los resultados más destacados. 


\section{Datos y metodología}

El objetivo inicial del estudio era dar cuenta de la popularidad del gabinete socialista durante todo su mandato, desde el inicio de 1983 hasta el primer trimestre de 1996, pero la escasez de datos para algunas de las variables relevantes entre 1983 y 1984 ha disminuido el período de estimación, por lo que ha quedado reducido a 1985-1996. La periodicidad de las series utilizadas es trimestral, puesto que algunos de los datos que se necesitan (concretamente, los relativos a la popularidad del Gobierno) no se publican mensualmente ${ }^{1}$.

Respecto a la variable dependiente, la literatura acostumbra a utilizar indistintamente como índices de popularidad, tanto la aprobación de la gestión del Gobierno como la intención de voto. Aunque en realidad estos dos indicadores están midiendo aspectos conceptualmente distintos (aprobación gubernamental no es realmente lo mismo que intención de voto) y se puede considerar que el primer indicador es un índice más preciso de popularidad que el segundo, la opción por uno u otro se debe, principalmente, a la disponibilidad y a la calidad de los datos.

En el caso español, no existe ninguna fuente que nos pueda proporcionar datos periódicos de aprobación de la gestión gubernamental con una frecuencia que se aproxime, ni tan sólo remotamente, a la trimestral. El Centro de Investigaciones Sociológicas (CIS) es la mejor fuente, pero, además del problema de la frecuencia ya mencionado, la formulación de sus cuestiones ha variado cinco veces en el período de referencia, lo que imposibilita considerar sus datos como una serie continua. Sí existen, sin embargo, series de intención de voto hacia el partido gubernamental, suministradas también por el CIS con una periodicidad que oscila entre uno y tres meses. Por lo tanto, en estas circunstancias, la mejor opción es utilizar como indicador de popularidad el porcentaje de intención de voto del partido socialista.

En cierto sentido, el indicador que aquí se utiliza (intención de voto) es más exigente que otros. De hecho, si los gobiernos se preocupan por su popularidad es, además de la satisfacción de sentirse apreciados, porque se trata de algo que puede generar votos a largo plazo. Así, modelando la intención de voto en lugar de la valoración de la gestión, se avanza un paso en la cadena causal y se adopta una posición más exigente en vez de una más confortable.

La variable dependiente es, pues, el porcentaje de intención de voto al partido socialista calculado teniendo en cuenta únicamente a las personas que expresan intención de voto hacia algún partido en concreto, quedando excluidos los indecisos. Se es consciente que ésta es también una práctica discutible, ya que así se da por sentado que la intención de voto de los indecisos se distribuye de la misma manera que en el resto de la muestra. Sin embargo, la estrategia alternativa (calcular el porcentaje sobre toda la muestra) también

1. Todos los datos utilizados en este estudio están a la libre disposición de los investigadores. Las peticiones pueden dirigirse al Institut de Ciències Polítiques i Socials o directamente a los autores. 
puede ponerse en tela de juicio, puesto que un aumento de indecisión puede aparecer como un descenso de la popularidad del Gobierno, y no siempre es así. Por lo tanto, la opción tomada es tan arbitraria como cualquier otra, pero ofrece una elegante ventaja: unos números que resultan creíbles como porcentajes de voto. En algunos trimestres para los que hubo más de una medición, se calculó la media aritmética de las observaciones disponibles. De esta manera, la serie de popularidad construida a partir de los datos de intención de voto del CIS consta de más de cincuenta observaciones.

Con respecto a las variables independientes, se debe señalar que se trabajó con un gran número de variables económicas y políticas que, teóricamente, podían afectar a los niveles de popularidad del Gobierno. Sin embargo, gran parte de ellas o bien resultaron ser no significativas, o bien lo eran pero su efecto desaparecía al controlar por terceras variables. En la sección 7 se da cuenta de todas las variables descartadas. Las que realmente entraron en el modelo obtenido al final del análisis son las siguientes:

- Desempleo, medido como el porcentaje de parados respecto a la población activa. Este dato es suministrado por la OCDE.

- Inflación, igual al nivel oficial de inflación que proporciona el Instituto Nacional de Estadística (INE). Concretamente, es el índice general en su versión interanual. Puesto que los datos oficiales sobre la inflación aparecen mensualmente, se obtuvo una media trimestral.

Siguiendo la práctica convencional, los niveles de desempleo e inflación fueron diferenciados hasta transformarlos en estacionarios. En ambos casos, ello implicó diferenciarlos por partida doble. Por esta razón, los coeficientes de regresión generados, por ejemplo, por la variable desempleo deben interpretarse como un incremento de popularidad producido por un incremento marginal de la aceleración del nivel de desempleo. Nótese que no deberían interpretarse como un incremento de popularidad producido por un incremento marginal en el nivel de desempleo, ni tampoco por un incremento marginal del crecimiento del nivel de desempleo. Lo mismo ocurre con la inflación.

- Expectativas económicas generales, que son medidas mediante las respuestas a una pregunta de encuesta sobre la situación económica esperada en el país durante el siguiente año. Su valor se ha tomado de las series económicas de la Unión Europea (UE), que miden las respuestas de la manera siguiente: mucho mejor, +1 ; mejor, $+1 / 2$; más o menos lo mismo, 0 ; peor, $-1 / 2$, y mucho peor, -1 . Esta ponderación también puede ser discutible, pero es la que proporciona directamente la UE, lo que impide cualquier adaptación.

Por otro lado, ya que la UE no proporciona datos sobre España hasta el año 1986, también se utilizaron los equivalentes funcionales de las preguntas de las encuestas del CIS para el período 1985-1986. Sin embargo, no fue posible disponer de datos para el período 1983-1984, ni de la UE ni del CIS, hecho que redujo en ocho puntos el período de estimación. Para combinar la información de las dos fuentes, las series del CIS fueron transformadas monótona- 
mente hasta hacerlas fluctuar en el mismo nivel que las principales series de la UE. La transformación lineal se estableció haciendo una regresión de las series del CIS respecto a las principales series de la UE. Finalmente, dado que los datos aparecían mensualmente, se calculó su media trimestral.

- Liderazgo, variable que intenta medir el carisma del presidente Felipe González. Los datos proceden de las encuestas del CIS, en las que se solicita a los ciudadanos que sitúen a los líderes políticos en una escala del 0 al 10 . El CIS proporciona la evaluación media para cada líder en cada momento. La variable de liderazgo se calculó como la diferencia entre la evaluación media de Felipe González y la evaluación media del correspondiente líder del Partido Popular en cada momento: Fraga hasta el año 1989, y Aznar a partir entonces.

Tanto la variable de expectativas económicas generales como la de liderazgo fueron diferenciadas una vez para convertirlas en estacionarias. Esto significa que los coeficientes de regresión generados, por ejemplo, por las expectativas económicas generales se deben de interpretar como el aumento de popularidad producido por un incremento marginal del crecimiento de las expectativas económicas generales (es decir, no por un incremento marginal de las expectativas económicas generales por ellas mismas). Lo mismo ocurre con el liderazgo.

Finalmente, aparecen otras tres variables que capturan los efectos de fenómenos políticos impredecibles y cuya justificación se detalla posteriormente, en la sección 6. Éstas son:

- Comunidad Europea, variable dicotómica que toma el valor 1 en el momento de entrada de España en la Comunidad Europea (cuarto trimestre de 1985) y 0 en los demás casos.

- Kuwait, variable dicotómica que toma el valor 1 cuando estalla la Guerra del Golfo (en el tercer trimestre de 1990) y 0 en los demás casos.

- Filesa, variable dicotómica que modela los efectos del llamado "caso Filesa» de financiación irregular del partido socialista. Toma valor 1 en el cuarto trimestre de 1993 y primero de 1994 y 0 en los demás casos.

El proceso seguido para modelar la popularidad se basó en la aplicación de la metodología de Hendry (1983). Ésta consiste en intentar estimar el modelo introduciendo todas las variables independientes que, en teoría, puedan influenciar la variante dependiente, junto con todos los retardos de las mismas que parezcan razonables. Como puede esperarse, se obtiene un modelo en el que la mayoría de los coeficientes de regresión no son estadísticamente significativos. En consecuencia, se excluyen las variables independientes asociadas a dichos coeficientes y vuelve a estimarse el nuevo modelo así obtenido, que es mucho más reducido. En el caso de que alguno de los nuevos coeficientes estimados resulte ser no significativo, debe reiterarse el proceso.

Además de la metodología Hendry, se aplicó también una estrategia complementaria basada en una observación variable a variable. En este caso, cada una de las variables independientes, con sus correspondientes retardos de dos 
o tres períodos, fue utilizada como predictor único de la variable dependiente. Ello generó una lista de variables y retardos de las mismas que demostraron tener una influencia autónoma en la popularidad. Tal como era de esperar, en esa lista estaban presentes más o menos las mismas variables que en el modelo final generado por el método anterior. Sin embargo, las pocas variables que figuraban en la lista, pero no en el modelo anterior, fueron reincorporadas en el modelo general, contrastándose si mantenían o no su significación estadística. Así se pudo confirmar o rechazar la solidez de su influencia. Esta rutina no cambió el modelo, pero fue útil para corroborar que el modelo final obtenido estaba bien especificado y que ninguna de las variables presumiblemente influyentes había sido omitida.

\section{Panorámica de resultados}

Siguiendo el método de estimación descrito en la sección anterior, se llegó al modelo final de popularidad para el Gobierno español durante el período socialista (exceptuando sus dos primeros años) que se muestra en la tabla 1.

Tal como puede observarse, todas las variables que aparecen en él, excepto la constante, son significativas, con niveles de significación siempre inferiores al nivel estándar del 0,05. Los signos de los coeficientes de las variables económicas son los esperados de acuerdo con la hipótesis de la responsabilidad: negativos para el desempleo y la inflación y positivos para las expectativas económicas generales. Los signos de los coeficientes de las variables políticas también son los esperados (exceptuando el de Kuwait, cuya interpretación se discute posteriormente): positivos para liderazgo, Comunidad Europea y Kuwait, y negativo para Filesa. Finalmente, los tests estadísticos estándares

Tabla 1. Intención de voto para el Partido Socialista (1985-1996)

\begin{tabular}{|c|c|c|}
\hline & Coeficiente & Significación \\
\hline $\begin{array}{l}\text { Componente estructural } \\
\text { Intención de voto } \\
\text { Constante }\end{array}$ & $\begin{array}{l}0,955 \\
1,71\end{array}$ & $\begin{array}{l}0,000 \\
0,423\end{array}$ \\
\hline $\begin{array}{l}\text { Componente económica } \\
\text { Desempleo }_{\mathrm{t}-3} \\
\text { Inflación }_{\mathrm{t}-2} \\
\text { Expectativas económicas generales }_{\mathrm{t}-1}\end{array}$ & $\begin{array}{r}-2,59 \\
-0,63 \\
0,16 \\
\end{array}$ & $\begin{array}{l}0,013 \\
0,036 \\
0,010 \\
\end{array}$ \\
\hline $\begin{array}{l}\text { Componente política } \\
\text { Liderazgo } \\
\text { Comunidad Europea } \\
\text { Kuwait } \\
\text { Filesa }\end{array}$ & $\begin{array}{r}2,78 \\
6,59 \\
6,59 \\
-4,13\end{array}$ & $\begin{array}{l}0,019 \\
0,006 \\
0,007 \\
0,004\end{array}$ \\
\hline
\end{tabular}

El modelo no presenta problemas al ser sometido a los tests estándares de correlación serial, forma funcional, normalidad y heteroscedasticidad. $\mathrm{R}^{2}$ ajustado $=0,93$; Estadístico Durbin Watson $=2,38$. 
indican que el modelo no presenta problemas de autocorrelación, heteroscedasticidad ni forma funcional, y explican en su conjunto un $93 \%$ de la variación total de la popularidad del Gobierno socialista durante el período analizado.

A continuación, se interpretan detalladamente estos resultados paso a paso: ante todo, la componente económica; en segundo lugar, la componente estructural, y, en tercer lugar, la componente política.

\section{Resultados para la componente económica}

La tabla 1 muestra el impacto que tiene el desempleo sobre la intención de voto para el partido en el gobierno: ésta disminuye en más del 2,5\% por cada incremento marginal de la aceleración de la tasa de desempleo. Por otro lado, cada incremento marginal de la aceleración de la tasa de inflación hace disminuir la intención de voto en dos tercios de punto. El hecho de que lo que cuenta no sean los niveles — ni su incremento-, sino la aceleración de las tasas, sugiere que los votantes españoles se acostumbraron fácilmente a las circunstancias económicas. Por ejemplo, incluso en un contexto de disminución del desempleo, se castigaba al Gobierno si las tasas, de repente, no bajaban tan rápidamente como en el momento anterior.

El desempleo tardó tres trimestres en afectar a la intención de voto, y la inflación tardó dos. Estos resultados parecen perfectamente comparables con los hallados en otros países.

Aún sabiendo que la relación entre la popularidad del Gobierno (se mida como se mida) y el desempleo o la inflación aparece frecuentemente, no deja de producir un cierto sentimiento de sorpresa. ¿Cómo pueden reaccionar los españoles frente a datos macroeconómicos si se sabe que sus conocimientos de macroeconomía son limitados (no es que se tenga nada personal contra los españoles: ¡comparten su débil conocimiento macroeconómico con el resto de la humanidad!)? O, dicho de otra manera, ¿castigaron realmente los españoles al Gobierno socialista porque sabían que la macroeconomía empeoraba? Como indica el profesor Sanders (2000), la respuesta a esta pregunta parece ser que los votantes realmente no necesitan tener gran erudición macroeconómica para percibir la tendencia general de las condiciones económicas. Los datos sobre la estabilidad de los coeficientes en el modelo estimado para España (de los que ahora trataremos) parecen apoyar esta idea.

¿Qué grado de estabilidad ofrece esta relación entre intención de voto, por un lado, y desempleo e inflación, por otro? En otras palabras, ¿bajó la intención de voto un $2,59 \%$ por cada incremento marginal en la aceleración del nivel de desempleo durante todo el periodo estudiado? ¿O bajó de manera más acusada en algunos momentos? Teniendo en mente esta cuestión, se realizaron dos tipos de análisis: regresiones recursivas y regresiones rodantes o móviles (rolling regressions). Las regresiones recursivas, básicamente, implican estimar repetidamente el modelo con períodos sucesivos de estimación que van aumentando en cada estimación. Por ejemplo, primero, se estima el modelo para el período 1985-1987; luego, se vuelve a estimar con una observación más, la del primer 
trimestre de 1988; y así sucesivamente. Ello significa que cada estimación prolonga el período en un trimestre hasta que se llega a estimar el modelo completo que figura en la tabla 1 . Lo interesante de este análisis es que puede apreciarse la estabilidad del coeficiente siguiendo simplemente los valores que toma el coeficiente en su trayectoria hacia su valor final. Los gráficos 1 y 2 muestran la regresión recursiva para los coeficientes de desempleo e inflación.

Por otro lado, la regresión rodante implica estimaciones sucesivas del modelo para períodos de una amplitud determinada, por ejemplo, de unos veinticinco puntos. El proceso es el siguiente: primero se estima el modelo con las primeras veinticinco observaciones. Luego se estima de nuevo el modelo eliminando la primera observación y añadiendo en su lugar la observación posterior al período de estimación utilizado anteriormente y así sucesivamente hasta llegar a incluir la última observación. Este método también produce sucesivas reestimaciones del modelo, pero el período utilizado en cada una de ellas siempre incluye la misma cantidad de observaciones. Este análisis sirve también para contrastar la estabilidad de los coeficientes al mostrar los que se obtienen en las estimaciones consecutivas. Los gráficos 3 y 4 presentan las estimaciones obtenidas mediante la regresión rodante para los coeficientes de desempleo e inflación.

Cada uno de los gráficos, tanto los correspondientes a regresiones recursivas como los correspondientes a regresiones rodantes, presentan tres líneas. La línea central corresponde al valor estimado para el coeficiente en cada período. Las líneas superior e inferior delimitan el margen de error de la estimación para una confianza del $95,5 \%$. Es decir, muestran el valor de la estimación más y menos dos veces el error típico de la misma. La interpretación que debe darse a estos gráficos es la siguiente: si las bandas de error quedan claramente por encima o por debajo de la línea del cero, se puede afirmar (con una confianza del $95,5 \%$ ) que el coeficiente estimado es distinto de cero, con signo positivo o negativo según sea el caso, y que, por consiguiente, la correspondiente variable es significativa durante todo el período; si, por el contrario, las bandas de error contienen al cero en algún período, no se puede afirmar que la variable sea significativa en ese período ${ }^{2}$.

Se interpretan ahora ambos análisis de estabilidad para cada una de las dos variables macroeconómicas. Los gráficos 1 y 3 muestran como el desempleo influyó cada vez menos en la intención de voto de los españoles. De hecho, hubo una época en que produjo una caída de la intención de voto cercana al $4 \%$, pero a principios de los años noventa redujo su impacto a menos del $3 \%$. Hacia 1994, el valor absoluto del coeficiente cayó hasta tal nivel que el margen de dos veces el error estándar traspasa el punto cero, lo que indica problemas de significación estadística. En resumen, el desempleo acarreó numerosas consecuencias en los años ochenta, pero fue perdiendo impacto electoral durante los años noventa.

2. Es normal (y carece de importancia) que en las regresiones recursivas las bandas de error al principio sean muy anchas, puesto que se han usado pocas observaciones en las estimaciones y, en consecuencia, los errores típicos son elevados. 


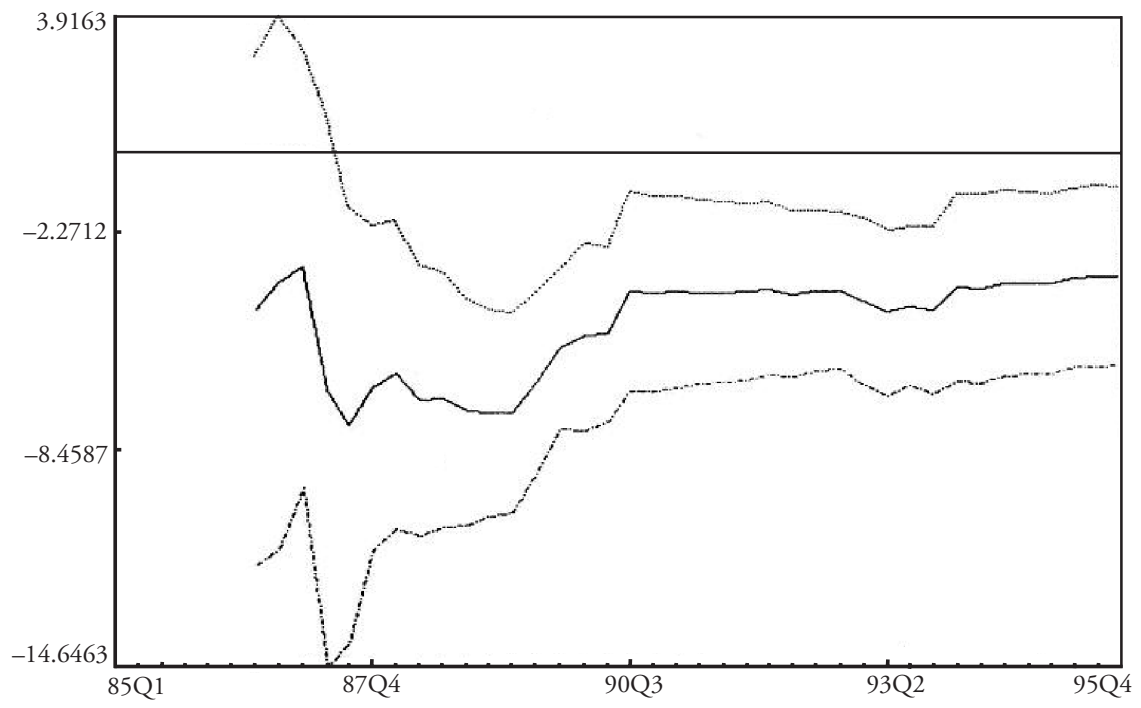

Gráfico 1. Regresión recursiva. Coeficientes para el desempleo y sus márgenes de error para una confianza del $95,5 \%$.

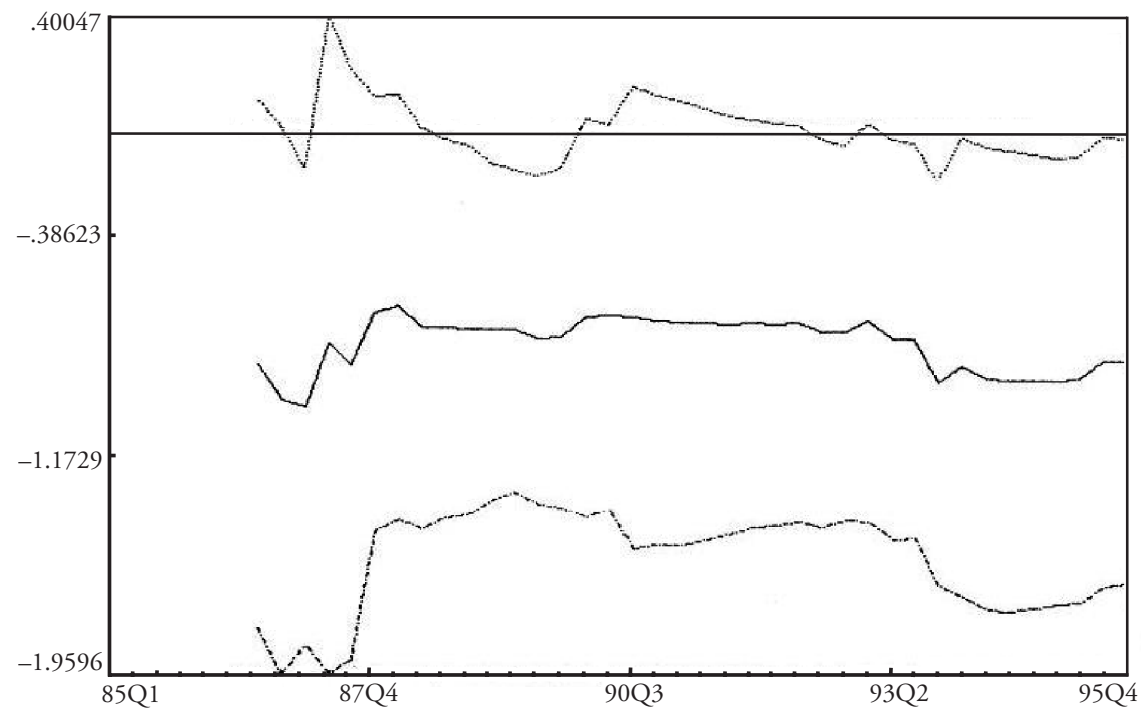

Gráfico 2. Regresión recursiva. Coeficientes para la inflación y sus márgenes de error para una confianza del $95,5 \%$. 


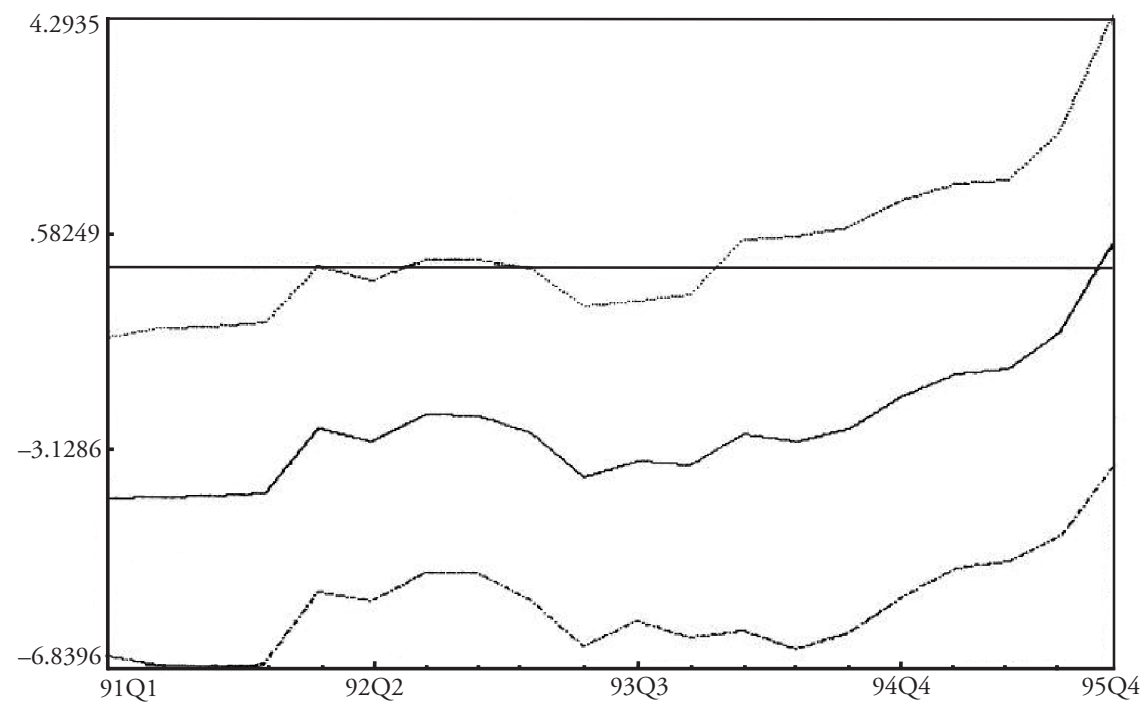

Gráfico 3. Regresión rodante. Coeficientes para el desempleo y sus márgenes de error para una confianza del 95,5\%.

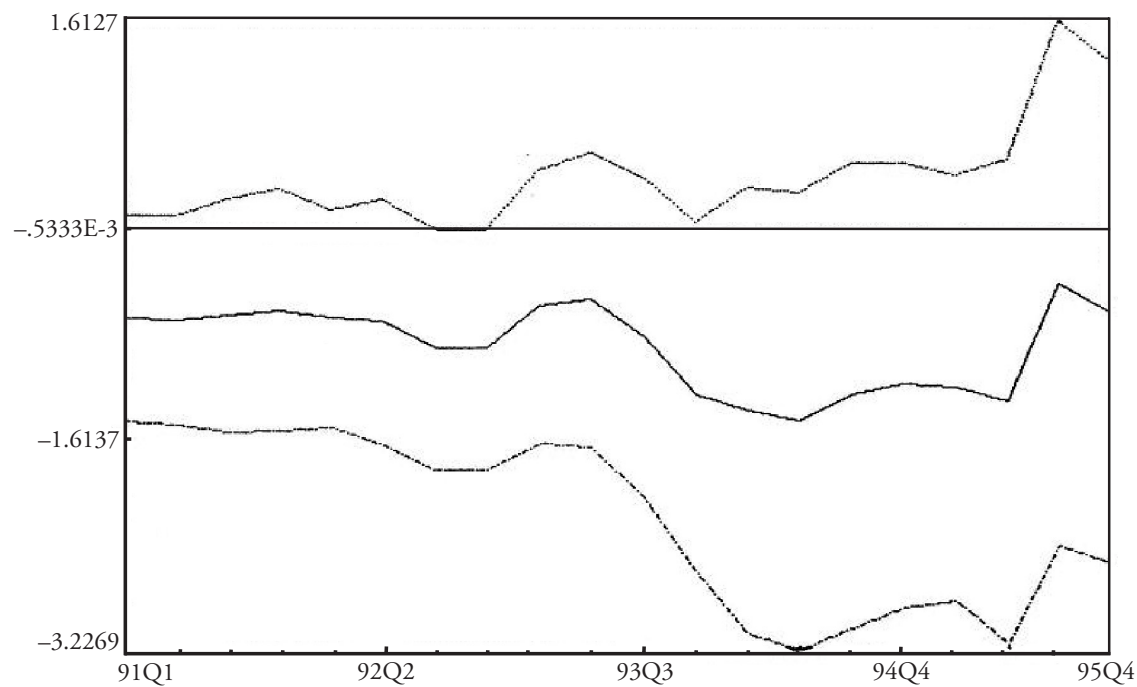

Gráfico 4. Regresión rodante. Coeficientes para la inflación y sus márgenes de error para una confianza del 95,5\%. 
Por otro lado, los gráficos 2 y 4 revelan el efecto contrario en referencia a la inflación. Al principio del período de análisis, la inflación tenía una influencia modesta sobre la intención de voto de los españoles. En los años noventa, el coeficiente cayó hasta casi alcanzar el -1, lo que muestra un aumento de su impacto, que fue máximo en torno a los años 1992 y 1993. Sin embargo, estas conclusiones deben tomarse con cautela, puesto que hay indicios de una significación estadística problemática en el gráfico 4, indicios que dependen de la técnica estadística y del período analizado ${ }^{3}$.

La existencia de dos períodos en los que las variables de desempleo e inflación parecen intercambiar su influencia pudiera ser la causa de que los coeficientes estimados para ellas sean significativos a un nivel $\mathrm{a}=0,05$ pero no lo sean para un nivel $a=0,01$. La significación observada $(0,013$ y 0,036 respectivamente) parece ser sólo una media entre dos períodos de distinta significación estadística.

En el aspecto sustantivo, para interpretar la existencia de dos períodos de distinta significación de las variables desempleo e inflación, debe tenerse en cuenta el cambio de agenda económica que tuvo lugar durante el período estudiado. En efecto, los años ochenta habían empezado con la elección de un gobierno socialista. Por primera vez en muchas décadas, los miembros de sindicatos formaban parte del Gobierno y, con el nivel más elevado de desempleo de Europa, el desempleo figuraba entre las principales preocupaciones de la agenda política. Sin embargo, esta situación cambió con el paso del tiempo. La adhesión a la Comunidad Europea y el principio del Mercado Único en el año 1986 comportaron un cambio en las prioridades económicas. Desde entonces y hasta la culminación del proceso en el año 1992, se empezó a considerar la inflación como el principal problema económico. Aquel año se firmó el Tratado de Maastricht y, paralelamente, se ratificó la inflación como el problema, con lo que el desempleo quedó relegado a una posición subordinada en la agenda económica. Este cambio de prioridades estaba claro en la agenda de las élites, pero el cambio de significación de las variables desempleo e inflación en la función de popularidad parece indicar que también se produjo en la agenda de los ciudadanos.

Una consecuencia colateral de dicho cambio de influencia entre desempleo e inflación alude a la posibilidad mencionada anteriormente de que los ciudadanos capten cual es la tendencia general de la situación económica. En efecto, según la interpretación dada, parece que los españoles mostraron un cambio de prioridades en su agenda económica. De ser así, ello implicaría que los ciudadanos tienen realmente una agenda económica, aunque sea imprecisa. Esta circunstancia, en cierto modo, avalaría la idea de que los votantes perciben efectivamente cual es la tendencia general de las condiciones económicas a pesar de tener escasos conocimientos sobre macroeconomía.

3. En la regresión rodante las estimaciones se realizan a partir de solamente 25 observaciones, con lo que los errores típicos son mayores que cuando se estima el modelo completo. 
La tabla 1 también muestra la presencia de una tercera variable en la componente económica de la función: las expectativas económicas generales. Cada incremento marginal en el aumento de expectativas comportó un incremento de 0,16 puntos en la intención de voto. Este coeficiente no mostró problemas de inestabilidad en las regresiones recursivas y rodantes y tampoco presenta problemas de significación estadística (sig = 0,01).

Se trata ahora de ubicar los resultados de las variables económicas de la función española en el debate académico internacional sobre las funciones de popularidad (véase el ya mencionado Bosch y otros, 1999, para una iniciación a estos debates). El hecho que las expectativas generales sobrepasen la influencia de las expectativas personales fácilmente situaría el caso español en el bando de los sociotrópicos, mientras que el hecho de que las expectativas generales sobrepasen la influencia de las retrospecciones generales, lo situaría en el sector de la prospectiva. Pese a reconocer el atractivo teórico de las cuestiones contenidas en dichos debates, los autores tienden a pensar que el hecho de que sea una u otra la variable que supere mejor los contrastes de significación estadística puede ser una cuestión meramente accidental. Por ejemplo, los datos para las cuatro variables no cubren exactamente los mismos períodos, lo que podría sesgar en buena medida el acierto relativo de las mismas.

\section{Resultados para la componente estructural}

Siguiendo una práctica convencional, se incluyó la variable dependiente retardada como una variable independiente. Esta especificación del modelo implica suponer que la intención de voto de un momento determinado depende de la intención de voto de que disfruta el Gobierno en el período anterior. Por esta razón, el coeficiente para dicha variable puede interpretarse como el porcentaje de la intención de voto que el Gobierno mantiene para el siguiente período: una especie de porcentaje básico del voto sobre el que influirían las restantes variables para hacerlo aumentar o disminuir más todavía. En razón de esta explicación, se suele llamar a dicho coeficiente tasa de depreciación. Se trata, de hecho, de la velocidad con la que el simple paso del tiempo erosiona la intención de voto de un período inicial. Puesto que el término velocidad de erosión sonaría más próximo a la geología que a la política, se ha optado por el término tasa de depreciación.

Dicha especificación, que nos había parecido una hipótesis razonable, resultó ser también empíricamente correcta. Como indica la tabla 1, el Gobierno socialista consiguió mantener casi el 96\% de su intención de voto en el período siguiente. Teniendo en cuenta que se utilizan datos trimestrales, se trata de una tasa de depreciación muy importante, más alta que cualquier otra de las mencionadas en la literatura existente. ¿Significa esto que los españoles mantienen la popularidad o impopularidad del Gobierno durante un largo período de tiempo, independientemente de las circunstancias del momento? $\mathrm{O}$, como dicen los expertos, ¿significa esto que los españoles consideran los hechos a largo plazo? Depende. Los análisis de estabilidad que reproducen los gráfi- 
cos 5 y 6 demuestran que dicha actitud sólo se manifiesta durante el último período de análisis, siendo, no obstante, suficiente para incrementar exageradamente la estimación general de la tasa de depreciación.

Los gráficos 5 y 6 muestran que la tasa de depreciación se mantiene bastante estable hasta el año 1993. Apenas fluctúa alrededor del 0,55\%, lo que representa una tasa de depreciación trimestral perfectamente estándar ${ }^{4}$. Sin embargo, el año 1993 comienza con un salto en la tasa de depreciación, que sube al $0,7 \%$, y después se produce un continuado aumento de la misma hasta 1994 , momento en que se aproxima a la unidad. Este modelo sugiere que los españoles preveían tan a corto plazo como el resto del electorado europeo hasta el año 1993, mientras que a partir de entonces ocurrió algo extraordinario que resituó todas las cosas.

Cualquier experto en política española estará de acuerdo con el hecho de que el período 1993-1995 fue, a todas luces, extraordinario. Se organizó una campaña mediática contra el Gobierno socialista desde el día siguiente a los sorprendentes resultados electorales de 1993 (Maravall, 1996: 19; Carol, Tarín y Martín de Pozuelo, 1998), cuando, contra todo pronóstico, el partido socialista volvió a ganar las elecciones generales Los titulares mencionaban escándalos cada día, las ofensas personales eran cada vez más desagradables y, de hecho, se recordaba diariamente a los electores los males de todo el mandato socialista. Según se desprende de las declaraciones del entonces director del periódico $A B C$, Luís María Ansón ${ }^{5}$, la campaña contribuyó, finalmente, al fracaso electoral del Gobierno socialista en marzo de 1996. Sin embargo, los datos sugieren que consiguió algo más: indujo a los españoles a aumentar su tasa de depreciación haciéndoles recordar los sucesos pasados durante un tiempo mucho más largo. De hecho, la tasa de depreciación cercana a uno sugiere que la campaña motivó que los españoles recordaran continuamente las condiciones anteriores.

Por esta razón, el criterio de los autores es que este salto en la tasa de depreciación no debería considerarse como una anomalía de la función, sino, precisamente, como la confirmación de su capacidad para detectar cambios en la atmósfera política.

El segundo elemento en la componente estructural es la constante de la ecuación. Si se hubiera estimado a través de una regresión estándar, dicha constante indicaría que el Gobierno socialista poseía una cuota mínima garantizada de voto del 1,7\%. Sin embargo, algunas peculiaridades metodológicas llevan a descartar esta interpretación. La inclusión de la variable dependiente

4. La pequeña alteración súbita hacia el tercer trimestre de 1990 se debe exclusivamente a un detalle técnico. Dado que estos análisis no admiten variables dicotómicas, se tuvo que excluir temporalmente una de ellas, situada en este período (véase sección 5), lo que comporta que la tasa de depreciación parece sufrir dicha ausencia, absorbiendo el efecto de esta variable dicotómica.

5. Recogidas en una entrevista realizada por Santiago Belloch y publicada en el semanario Tiempo el 16 de febrero de 1998. 


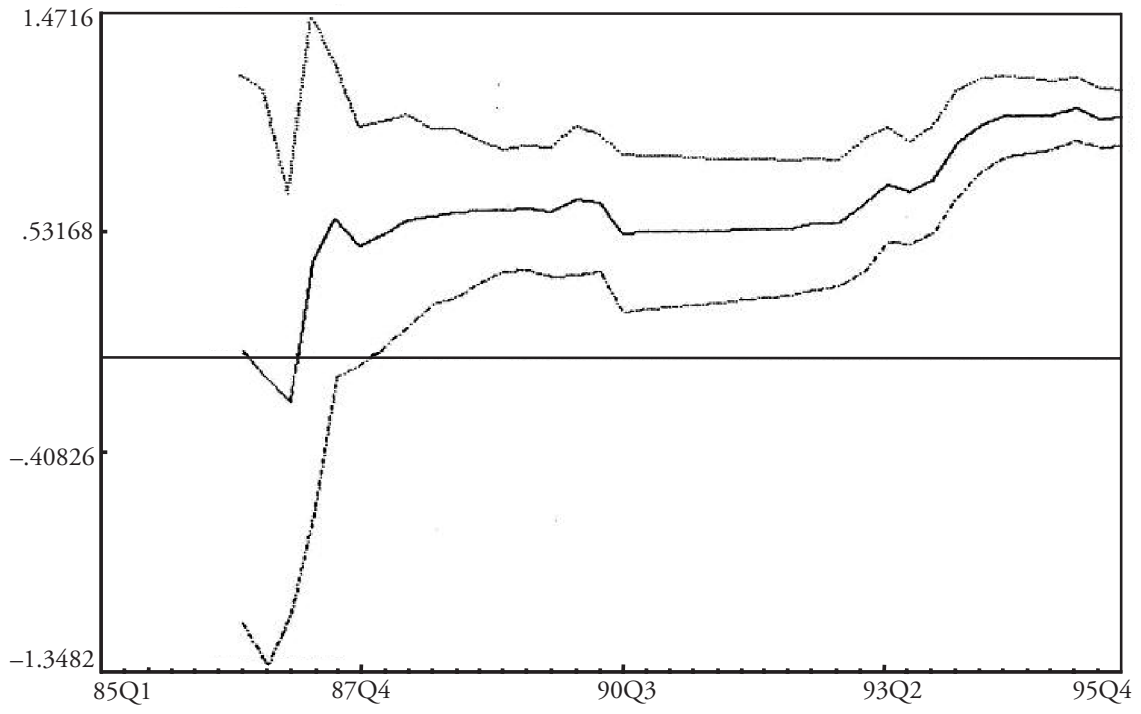

Gráfico 5. Regresión recursiva. Coeficientes para la tasa de descuento y sus márgenes de error para una confianza del $95,5 \%$.

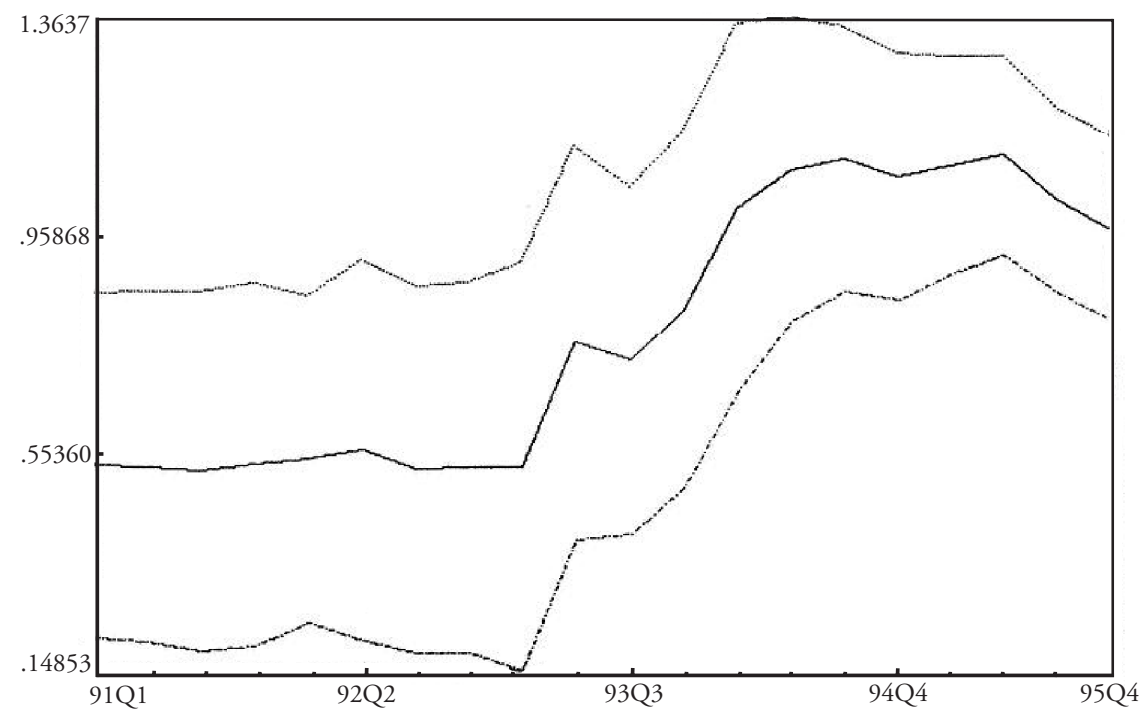

Gráfico 6. Regresión rodante. Coeficientes para la tasa de descuento y sus márgenes de error para una confianza del $95,5 \%$. 
retardada significa que, en cada momento, la estimación de la intención de voto arranca de un valor positivo y relativamente alto, valor que equivale a la intención de voto del momento anterior multiplicado por la tasa de depreciación. Este elemento proporciona una base (una cuota mínima garantizada de voto) y, por tanto, la presencia de la constante se convierte, de alguna manera, en redundante. La inclusión de la variable dependiente retardada también significa que no se está evaluando la intención de voto hacia el Gobierno, sino que se consideran las variaciones de la intención de voto, lo que reduce enormemente la necesidad de una constante. En definitiva, dadas las características del modelo, no es de extrañar que su constante muestre serios problemas de significación estadística.

\section{Resultados para la componente política}

Si hubiera que estimar el modelo únicamente con las dos componentes mencionadas hasta ahora, la regresión produciría unos valores predichos para la intención de voto y éstos diferirían claramente de los valores reales en tres momentos específicos. El gráfico 7 muestra la diferencia entre los valores predichos y los reales (es decir, los residuos) de este tipo de modelo, junto con la banda de error de más y menos un error estándar a partir del cero.

Gracias a él, se observa claramente que algo excepcional ocurrió durante el cuarto trimestre de 1985, cuando la intención de voto real superaba en más

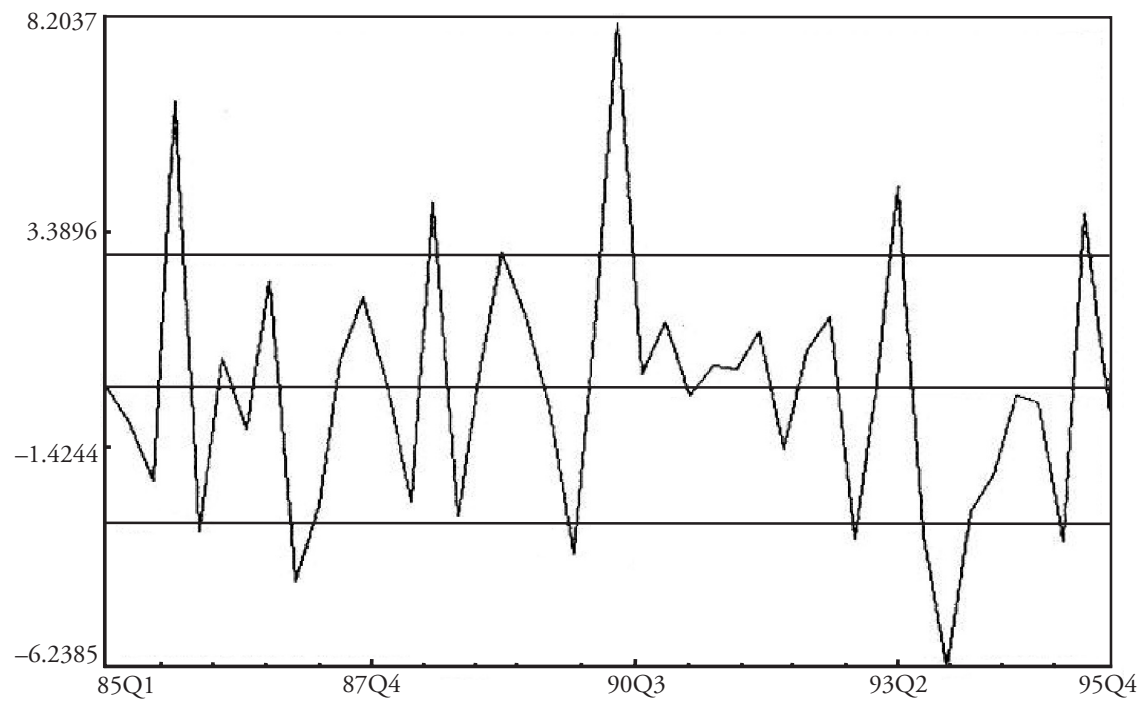

Gráfico 7. Residuos del modelo sin incluir variables dicotómicas y bandas a un error estándar del cero. 
de seis puntos el valor predicho. También ocurrió algo en el tercer trimestre de 1990, con una desviación de ocho puntos. Sucede también algo curioso con las predicciones del cuarto trimestre de 1993 y el primer trimestre de 1994, cuando los valores reales invariablemente transcurren por debajo de lo previsto.

No es necesario acudir a una hemeroteca para descubrir la razón de la extraordinaria popularidad del Gobierno en las encuestas del cuarto trimestre de 1985. Se acababa de firmar la entrada de España en la Comunidad Europea, lo que se consideró un logro histórico. Por tanto, resulta normal que los ciudadanos recompensaran al Gobierno con una intención de voto más elevada, del mismo modo que los ciudadanos tienden a recompensar, por ejemplo, las reducciones del desempleo. De acuerdo con la práctica habitual, se introdujo este hecho en la ecuación, modelándolo a través de una variable dicotómica que toma el valor 1 durante el cuarto trimestre de 1985 y el valor 0 durante el resto del período observado. Por lo tanto, el coeficiente de la variable dicotómica Comunidad Europea expresa el repentino salto en la intención de voto que obtuvo el Gobierno a raíz de tal acontecimiento. Así, la firma del tratado de adhesión hubiera proporcionado un 6,59\% de votos para el Partido Socialista si se hubieran celebrado elecciones inmediatamente después. Este efecto decayó con una tasa de depreciación del $0,55 \%$, por lo que en el momento de las elecciones generales de 1986 contribuyó con 2,4 puntos a la victoria socialista. El efecto de la Comunidad Europea siguió disminuyendo sin pausa y, a finales de 1986, se transformó en imperceptible (inferior al $1 \%)$.

Resulta más difícil de explicar el súbito sobresalto del tercer trimestre de 1990. Esa época coincidió con la Guerra del Golfo. La introducción de una variable dicotómica en el modelo en este punto muestra claramente que la guerra hizo aumentar en un 6,56\% la intención de voto hacia el Gobierno. Si estuviéramos en Gran Bretaña o en Estados Unidos, se podría aceptar que la participación activa del Gobierno en la guerra (uso de bases militares, participación del ejército, etc.) hiciera aumentar la popularidad del mismo, pero estamos analizando el caso de España, y ciertamente los españoles no saltaron de gozo con la guerra. Incluso cuando los datos nos sugirieron dicha posibilidad, los autores decidimos no dar crédito a esa interpretación. La respuesta a la incógnita comienza con la confirmación de que, incluso si los residuos muestran un extraordinario repunte durante este trimestre, el repunte de la intención real de voto es más moderado. De hecho, los residuos insólitamente altos son generados sobre todo por la fuerte caída de la intención de voto prevista. Y este fuerte descuento deriva de la caída de las expectativas económicas generales. Por lo tanto, la historia parece ser la siguiente: la guerra produjo una caída súbita de las expectativas económicas generales; en circunstancias normales, esto produciría un descenso en la intención de voto, pero no sucedió así (probablemente porque los ciudadanos no recriminaron al Gobierno las malas noticias) y la intención de voto se mantuvo estable o incluso aumentó un poco; esta estabilidad inesperada provocó un desajuste entre los valores reales 
y los previstos. Por esta razón, la variable dicotómica denominada Kuwait sólo significa que no se dispone de otros medios estadísticos para dar cuenta del elevado residuo. Resumiendo, la guerra no hizo aumentar demasiado la intención de voto hacia el Gobierno, pero tampoco hizo que descendiera, incluso cuando hubiera podido hacerlo en un $6,56 \%$.

El efecto de Kuwait disminuyó a una tasa de depreciación del 0,55\%, lo que significa que en el tercer trimestre de 1991 se volvió imperceptible. Dado que hasta 1993 no se convocaron elecciones, Kuwait no intervino en los resultados electorales en modo alguno.

Se ha introducido la última variable dicotómica en el modelo para explicar los residuos negativos destacables en el cuarto trimestre de 1993 y el primero de 1994. Durante este período, el Partido Socialista se vio inmerso en un gran escándalo de financiación irregular que se convirtió en el más notorio episodio de corrupción de la reciente historia de España. Fue conocido bajo el nombre de escándalo Filesa, que es el nombre de una de las empresas ficticias que desviaron fondos irregulares hacia el Partido Socialista. ¿¿Durante cuánto tiempo afectó este escándalo al Gobierno y, por lo tanto, cuáles son los períodos en los que la variable toma el valor 1? Eso es algo que no está claro. De hecho, Filesa constituyó una serie de escándalos consecutivos que salieron a la luz a lo largo de varios meses, lo que justifica cualquier especificación entre uno y tres trimestres. En estas circunstancias, se tomó un criterio empírico, utilizando la que produjera mejores estimaciones estadísticas. Como resultado, se optó por dar el valor de 1 para el cuarto trimestre de 1993 y el primero de 1994 y el valor de 0 para el resto del período observado.

Con esta definición, el coeficiente de la variable dicotómica Filesa indica el coste electoral trimestral del escándalo Filesa. Durante el cuarto trimestre de 1993, dicho coste fue del 4,13\%; mientras que en el primer trimestre de 1994 el coste fue del 4,13\% más el coste depreciado del efecto durante el trimestre anterior (es decir, 4,13\% + $(4,13 \% \times 0,955)=8,074 \%)$. En el momento de mayor coste electoral, durante el primer trimestre de 1994, el Gobierno fue penalizado con ocho puntos. Puesto que la tasa de depreciación de aquel período ya estaba en torno a la unidad, este coste se transfirió casi totalmente a las siguientes elecciones generales en marzo de 1996. Incluso después de tanto tiempo, el caso Filesa costó 5,57 puntos al Gobierno socialista. Haciendo una simple operación aritmética, esto significa que los socialistas perdieron nada menos que 1.381.684 votos por culpa de Filesa, lo que demuestra que probablemente se trataba del peor negocio en la historia del Partido Socialista.

La última variable introducida en el modelo es el liderazgo. Como se ha indicado en la sección 2, se intentó medir el carisma de Felipe González, comparándolo con los sucesivos líderes del Partido Popular. Albergamos serias dudas respecto a cualquier explicación de la popularidad del Gobierno que tome, como variable explicativa, la popularidad de su presidente. De hecho, opinamos que la explicación podía ser un tanto tautológica. Ésta es la razón por la que no introdujimos la variable de liderazgo en el modelo hasta que éste no estuvo estimado, pulido y reestimado. Finalmente, se introdujo dicha varia- 
ble pero con la decisión de que, si tenía como efecto desviar en parte el modelo estimado anteriormente, se conservaría el modelo anterior.

Sin embargo, los resultados fueron sorprendentes y el modelo se mantuvo adecuadamente. Los niveles de significación no se deterioraron en absoluto y los coeficientes no variaron demasiado. Así pues, se decidió mantener en él esta variable adicional. El coeficiente de liderazgo sugiere que la intención de voto del Gobierno aumentó en un 2,78\% por cada aumento marginal en el incremento de la distancia entre los dos líderes. Lo que confirma hasta cierto punto la idea, bastante extendida en la política española, que la larga duración del Gobierno socialista sólo fue posible gracias a la gran diferencia de carisma entre Felipe González y los sucesivos líderes de la oposición. Sólo cuando el Partido Popular promocionó un líder «elegible» fue posible un cambio en el Gobierno.

\section{Variables descartadas}

Tal como se ha apuntado anteriormente, durante el proceso de estimación del modelo se utilizaron otras variables macroeconómicas que fueron desestimadas por distintos motivos. Algunas de ellas porque no mostraron ninguna influencia estadísticamente significativa sobre la intención de voto. Se trata del producto interior bruto (medido interanualmente, con magnitudes nominales y reales, por el INE) y del consumo privado (cuyos datos son también suministrados por el INE). Las demás, porque, aunque mostraron tener alguna influencia sobre la intención de voto, presentaron otro tipo de problemas. Se trata de los tipos de interés (tasas trimestrales estándares, en magnitudes nominales y reales, suministradas por el Banco de España), el tipo de cambio (tipo nominal efectivo de la peseta frente a las monedas de los estados miembros de la UE, también suministrado por el Banco de España), los impuestos sobre la renta (volumen recaudado per cápita, datos suministrados por el INE) y el impuesto sobre el valor añadido (diversas especificaciones para los tipos del IVA, para el momento de aplicación del IVA, etc.).

El impuesto sobre el valor añadido y el impuesto sobre la renta, tomados por separado, demostraron tener cierta influencia negativa sobre la intención de voto, pero la significación estadística de dichos efectos desapareció drásticamente cuando fueron introducidas en el conjunto del modelo.

Por otro lado, el tipo de cambio demostró tener alguna influencia, tanto si se introducía aisladamente como si se añadía al conjunto del modelo. En ese caso, el coeficiente era de un 0,35 positivo y su significación, cercana al 0,05 $(\mathrm{a}=0,067)$. Sin embargo, el problema real por el cual se decidió descartarla fue que, al introducir el tipo de cambio en el modelo, éste presentaba problemas de autocorrelación y heteroscedasticidad, según se deducía de los correspondientes tests estadísticos. El caso de los tipos de interés real es especialmente interesante. Esta variable sólo demostró tener una cierta influencia sobre la intención de voto cuando se introducía acompañada de alguno de sus retardos. El problema es que las variables retardadas que la acompañabann en nin- 
gún caso eran significativas. Y si se eliminaban, la variable no retardada perdía toda su significación estadística. En cualquier caso, tampoco mostraban grandes resultados cuando, en vez de tratarse aisladamente, se incluían en el conjunto del modelo. El coeficiente era entonces del -0,6, y la significación, del 0,015 , pero los tests indicaban problemas de correlación serial y la tasa de depreciación aumentaba hasta la unidad. En estas circunstancias, se decidió prescindir también de esta variable.

Por lo que respecta a medidas subjetivas de la economía, las variables retrospectivas no demostraron tener ninguna relación importante con la intención de voto, pero las prospectivas sí. Concretamente, la variable de las expectativas personales, una vez diferenciada y retardada tres períodos, resultó ser estadísticamente significativa, con un una significación del 0,031. El problema estriba en el hecho de que la variable de las expectativas generales funcionaba mejor que ésta cuando ambas se introducían conjuntamente en el modelo general. Y lo mismo ocurrió con un interesante índice suministrado por el Ministerio de Economía que reúne varias medidas de análisis de la confianza en la evolución de la economía.

\section{Conclusiones}

Según se desprende del análisis realizado, las elevadas tasas de desempleo que afectaron a España fueron uno de los factores que más dañaron la popularidad de los diversos gobiernos socialistas presididos por Felipe González entre 1985 y 1993. Durante este período, las estimaciones indican que por cada punto de aumento en la tasa de aceleración del paro, la popularidad del Gobierno descendió en 2,5 puntos. La inflación también afectó negativamente al Gobierno, si bien en menor medida: por cada punto de aumento en la tasa de aceleración de la inflación, su popularidad disminuyó en dos tercios de punto. Se debe insistir en que no fueron los niveles de desempleo e inflación, ni tan solo sus respectivos crecimientos, sino la velocidad de esos crecimientos (es decir, la aceleración del desempleo y de la inflación) los que dañaron al Gobierno.

Un aspecto destacable es que ambas variables de tipo macroeconómico actuaron con retardos temporales relativamente importantes: tres trimestres en el caso de la tasa de desempleo y dos trimestres en el caso de la tasa de inflación. Si bien es habitual que estas variables actúen con una cierta demora, los resultados sitúan el caso de España en el grupo de aquéllos donde las variables macroeconómicas actúan con un retardo más elevado. Otro aspecto interesante que se desprende del análisis es que el impacto del desempleo y de la inflación en la popularidad no fue el mismo durante todo el período socialista. Probablemente debido a un cambio de prioridades en la agenda económica del Gobierno (pero que fue percibido también por los ciudadanos), el impacto del desempleo fue más claro durante la primera etapa de mandato socialista que en la segunda, y ocurrió lo contrario con el tema de la inflación. 
Las percepciones subjetivas también tuvieron un fuerte impacto en la popularidad gubernamental. En concreto, por cada punto de mejora de las expectativas sobre el estado general de la economía para el país para el próximo año, la popularidad del Gobierno creció en un sexto de punto. El efecto de las percepciones es, sin embargo, mucho más rápido que el de la economía objetiva, puesto que esta variable actuó con una demora de un solo trimestre.

El indiscutible carisma de Felipe González fue otro de los factores que incidieron en la popularidad del Gobierno. Por cada punto de diferencia entre la valoración del presidente y la del líder de la oposición, la popularidad del Gobierno mejoró en casi tres puntos.

Los efectos de las variables económicas se depreciaron durante todo el período con una tasa media de 0,95 , que es muy alta si se la compara con los países del entorno. Sin embargo, este valor parece ser el resultado de dos etapas distintas. Durante la primera, la tasa de depreciación se situó en valores absolutamente normales, pero, durante la segunda, se elevó hasta valores cercanos a la unidad. Ello indica que durante los últimos años de gobierno socialista se instauró una fuerte inercia en su popularidad (o más bien, impopularidad). La conjetura más plausible es que ello fuera debido a la fuerte acción de ciertos medios de comunicación que explotaron exhaustivamente una serie de escándalos.

Finalmente, existen tres fenómenos políticos puntuales que afectaron también a la popularidad del Gobierno. Se trata de la firma del tratado de adhesión a la CE, de la guerra de Kuwait y de la explosión del escándalo Filesa. Los dos primeros fenómenos tuvieron un efecto positivo claramente reflejado en las series de popularidad, mientras que el tercero tuvo un claro efecto negativo que fue, en parte, responsable de la derrota del partido socilista en las elecciones generales de 1996 .

\section{Bibliografía}

Amor Bravo, Elías M. (1985). «El ciclo político de los negocios y su referencia al caso español (1976-1985)». Información Comercial Española, 2.015: 4259-4268.

- (1987). «Modelos político-económicos en España». Revista del Instituto de Estudios Económicos, 2.

- (1990). «Un modelo político-económicos de interacción a largo plazo: el caso de las infraestructuras" Revista del Instituto de Estudios Económicos, 4.

ANDERSON, Christopher (1995). Blaming the Government. Citizens and the Economy in Five European Democracies. Londres: Sharpe.

BARREIRO, Belén (1999). «Justificaciones, responsabilidades y cumplimientos de promesas electorales». Revista Española de Ciencia Política, 1, p. 149-169.

Bosch, Agustí; DíAZ, Aida; RiBA, Clara (1999). «Las funciones de popularidad (1): estado de la cuestión y principales debates». Revista Española de Investigaciones Sociológicas, 85(1), p. 171-197.

Carol, Màrius; TARÍN, Santiago; Martín de POzuelo, E. (1998). «Escenarios para un golpe de papel. Cómo se gestó y desarrolló el plan que puso al límite la estabilidad del Estado». La Vanguardia, revista de 22 de febrero de 1998, p. 2-4. 
Clarke, H.D.; Steward, M. (1995). «Economic Evaluations, Prime Ministerial Approval and Governing Party Support: Rival Models Reconsidered». British Journal of Political Science, 25, p. 145-170.

DíAZ, Aida (1999). Evolució de la popularitat del Govern a Catalunya: la influència de la conjuntura econòmica i dels esdeveniments polítics. Universitat Autònoma de Barcelona. Tesis de doctorado.

DíaZ, Aida; Riba, Clara (1999). "Catalan Government Popularity. An Example of Economic Effects on Sub-national Government Support». Economics and Business Working Paper (UPF), 406, p. 1-31.

Downs, Anthony (1957). An Economic Theory of Democracy. Nueva York, Harper y Row. Traducción castellana: Teoría económica de la Democracia, Madrid: Aguilar, 1973.

FioRINA, M. P. (1981). Retrospective Voting in American National Elections. New Haven: Yale University Press.

Fraile, Marta (2001). Does the Economy enter the ballot-box? A study of the Spanish voters' decisions. Colección Tesis Doctorales/28. Madrid: Instituto Juan March de Estudios e Investigaciones.

- (2002a). "The retrospective voter in Spain during the 1990s». En DoruSSEN, Han; TAYLOR, Michael (ed.). Economic Voting. Londres: Routledge, p. 284-302.

- (2002b). «El voto económico en las elecciones de 1996 y 2000. Una comparación». Revista Española de Ciencia Política, 6, p. 129-151.

Goodhart, C.A.E.; Bhansali, R.J. (1970). «Political Economy». Political Studies, 18 , p. 43-106.

HendrY, D.F. (1983). «Econometric Modeling: The Consumption Function in Retrospect». Scottish Journal of Political Economy, 30, p. 193-229.

HibBS, D.A.JR. (1982). «On the Demand for Economic Outcomes: Macroeconomic Outcomes and Mass Political Support in the United States, Great Britain and Germany». Journal of Politics, 44, p. 426-462.

KINDER, Donald R.; KIEWEIT, Roderick D. (1981). «Sociotropic Politics: The American Case». British Journal of Political Science, II(2), p. 129-161.

KRAMER, Gerald H. (1971). «Short-term Fluctuations in U.S. Voting Behavior, 18961964». American Political Science Review, 65, p. 131-143.

LEWIS-BeCK, M.S. (1988). Economics and Elections: The Major Western Democracies. Ann Arbor, Michigan University Press.

MANCHA NAVARRO, Tomás (1993). Economía y votos en España. Madrid: Instituto de Estudios Económicos.

MaraVAll, José María (1996). «Accountability and Manipulation». Working Paper del Centro de Estudios Avanzados en Ciencias Sociales, 92.

MaraVAlL, José María; PrZEWORSKI, Adam (1999). «Reacciones políticas a la economía». Revista Española de Investigaciones Sociológicas, 87, p. 11-52.

MuelLER, John E. (1970). «Presidential Popularity from Truman to Johnson». American Political Science Review, 64, p. 18-34.

Nannestad, Peter; Paldam, Martin (1994). «The VP-function: A Survey of the Literature on Vote and Popularity Functions after 25 Years». Public Choice, 79, p. 213-245.

- (1997). «From the Pocketbook of the Welfare Man: A Pooled Cross-Section Study of Economic Voting in Denmark, 1986-92». British Journal of Political Science, 27, p. 119-137.

Norpoth, H.; LeWIs-Beck, M.; Lafay, J.-D. (eds.) (1991). Economics and Politics: The calculus of Support. Ann Arbor: University of Michigan Press. 
RibA, Clara; DíAZ, Aida (2002). «Economic Voting in Sub-national Government. Catalonian Evidence». En DorUsSEn, Han; TAYLOR, Michael (ed.). Economic Voting. Londres: Routledge, p. 173-199.

SÁNCHEZ CuenCA, Ignacio; BARREIRO, Belén (2000). Los efectos de la acción de gobierno en el voto durante la etapa socialista (1982-1996). Madrid: Centro de Investigaciones Sociológicas.

SANDERS, David (2000). «The Real Economy and the Perceived Economy in Popularity Functions: How Much do Voters Need to Know? A Study of British Data, 197497». Electoral Studies, 19, p. 275-294.

SANDERS, David; WARD, Hugh; MARSH, David (1987). "Government Popularity and the Falklands War: A Reassessment». British Journal of Political Science, 17(3), p. 281-313.

SCHUMPETER, Joseph (1945). Capitalism, Socialism and Democracy. Nueva York: Harper. 